\title{
An Examination of the Helium Vapor-Pressure Scale of Temperature Using a Magnetic Thermometer ${ }^{1}$
}

\author{
E. Ambler and R. P. Hudson
}

\begin{abstract}
The variation of the mutual inductance of two coils surrounding a paramagnetic crystal has been measured as a function of the saturation vapor pressure of helium in the range $1.3^{\circ}$ to $4.2^{\circ} \mathrm{K}$. The fact that this quantity should vary inversely as the absolute temperature has been made use of to investigate the consistency of two recently proposed vapor-pressure temperature scales. The results suggest errors above $2^{\circ} \mathrm{K}$ in the empirical equation proposed by Clement, Logan, and Gaffney (in contrast to the experiences of Erickson and Roberts with a magnetic thermometer) and are in closer accord with the thermodynamic calculation of Van Dijk and Durieux.
\end{abstract}

\section{Introduction}

All practical thermometry in the "liquid-helium region" (that is, between $1^{\circ}$ and $5.2^{\circ} \mathrm{K}$ ) is dependent, directly or indirectly, upon a knowledge of the relation between the saturation vapor pressure of helium and the absolute temperature. Many investigators measure directly the pressure over the liquid-helium cryostat and derive $T$ from $p$ - $T$ tables; others use the same procedure to calibrate a resistance thermometer or magnetic thermometer, etc. In this type of vapor-pressure measurement the accepted practice is to apply a depth correction ("hydrostatic head") to the measured value of $p$; this correction is, however, of somewhat doubtful validity and can be avoided by measuring the pressure in a vapor-pressure bulb that is in thermal equilibrium with the material under investigation (effectively zero immersion).

The $p$ - $T$ tables in general use at the present time comprise the "1948 scale" [1] ${ }^{2}$ and are based on the work of Schmidt and Keesom [2], Bleaney and Simon [3], and Kamerlingh Onnes and Weber [4]. Possible errors in the 1948 scale were admitted at the time of its preparation, notably in the $1.3^{\circ}$ to $2.2^{\circ} \mathrm{K}$ region from consideration of the helium isotherm measurements of Kistemaker [5], and between the normal boiling point and the critical point due to the sparseness of the experimental data upon which the scale was based in the latter region. The investigations of Erickson and Roberts [6] with a magnetic thermometer for the region $1^{\circ}$ to $4.2^{\circ} \mathrm{K}$ and those of Berman and Swenson [7] above $4.2^{\circ} \mathrm{K}$ with a gas thermometer provided a strong basis for a revision of the 1948 scale, and an empirical formula was developed by Clement and coworkers [8] from which a $p-T$ table could be conveniently calculated to any desired precision, and which fitted the new data to within $0.002 \mathrm{deg}$ throughout the entire range. A summary of the situation obtaining in October 1954, prior to the development of the Clement formula and the publication of the supporting evidence obtained by Corak et al. [9] from calorimetric work and by

\footnotetext{
A brief account of this work was presented at the Fourth International Conference on Low Temperature Physies, Paris, August 30 to September 8, 1955.

2 Figures in brackets indicate the literature references at the end of this paper.
}

Keller [10] from $\mathrm{He}^{4}$ and $\mathrm{He}^{3}$ isotherm data, has been given by Hudson [11]. The latter report made reference to some preliminary magnetic thermometer investigations by Hudson and de Klerk (unpublished) that were in qualitative agreement with the findings of Erickson and Roberts [6] but suggested somewhat larger errors in the 1948 scale in the region of $3.5^{\circ} \mathrm{K}$. These measurements have now been extended, using improved apparatus, and the results provide the subject of the present report. While this work was in progress, Keesom and Pearlman [12] reported that some anomalies in their calorimetric data could be removed upon reevaluating their data in terms of the Clement equation.

In summary, the evidence available in mid-1955 strongly supported the validity of the Clement equation. A revision of the 1948 scale, long overdue, therefore seemed feasible and the time opportune with the approach of the Fourth International Conference on Low Temperature Physies in Paris in September 1955. During this conference, however, the results of a new thermodynamic calculation of the $p-T$ relation, which differs by several millidegrees from the Clement equation below $1.5^{\circ} \mathrm{K}$ and above $2.2^{\circ} \mathrm{K}$, were announced by Van Dijk and Durieux. ${ }^{3}$

The first part of section 4 of this paper deals with an analysis of our data in terms of the Clement equation. In section 4.2 the same data are reanalyzed in terms of the Van Dijk-Durieux table.

\section{Apparatus}

To reduce uncertainties in the measurement of $p$, the saturation vapor pressure, occasioned by measurement of the bath-pressure and application of the hydrostatic-head correction, a vapor-pressure bulb was employed. A series of measurements was performed with the apparatus shown in figure 1, a.

The magnetic thermometer comprised a paramagnetic salt specimen, A (a 1-in. sphere ground from a large crystal of chromic methylammonium alum $)^{4}$

${ }^{3} \mathrm{H}$. van Dijk and M. Durieux, paper presented at the Fourth International Conference on Low Temperature Physics, Paris Aug. 30 to Sept. 8, 1955. We are indebted to Dr. van Dijk for kindly providing us with advance information on this calculation.

4 The choice of salt was based upon the requirements: Reproducible behavior, small crystal field splitting and dipole-dipole interaction, absence of direct exchange interaction, and ease of grow th of large crystals. 


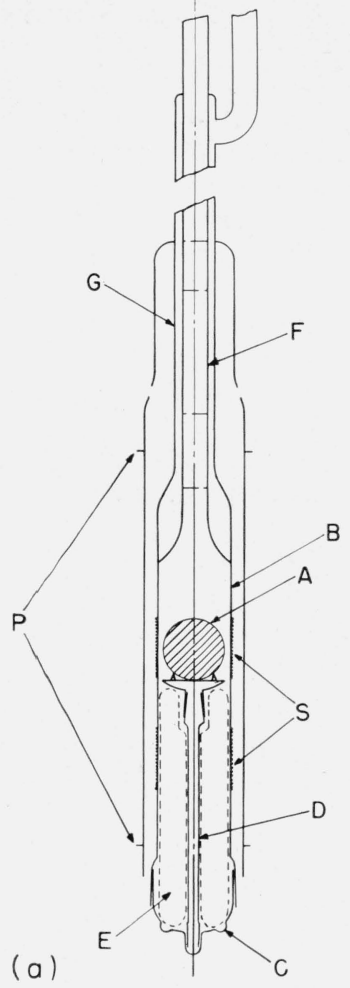

(b)

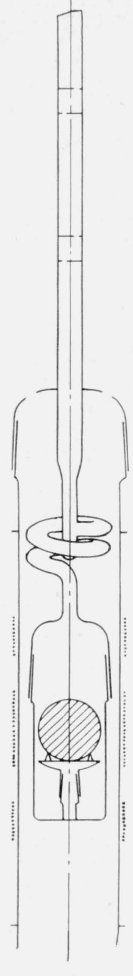

F Figure 1. Vapor-pressure bulb and magnetic thermometer.

' a, First apparatus, used in June 1955 measurements; b, modification as used in August 1955 measurements. For explanation of symbols, see text.

located within a mutual inductance, P-S, which was wound, in a rigid assembly, on concentric glass tubes. The latter was connected to an a-c mutual-inductance bridge [13]. For convenience of removal and inspection of the crystal, the bulb, B, was extended to project beyond the coil windings, and closed at the bottom by a ground-joint plug, C. A pedestal, D, supported the crystal, and the volume of the bulb was reduced by means of a hollow glass "filler", E. The tube, F, connected the bulb to the manometer system and was protected by a vacuum jacket, G. Sealed-in glass disks, each perforated by one small hole (about $2 \mathrm{~mm}$ in diameter), provided radiation shielding, and $\mathrm{F}$ and $\mathrm{B}$ were silvered internally.

The purpose of the jacket, G, was to avoid a "cold spot" on F: Due to the hydrostatic head effect, the liquid helium in the surrounding bath is coldest at the surface and, if the vapor in $\mathrm{F}$ comes to thermal equilibrium with the bath liquid at this point, the pressure indicated on the manometers will be that corresponding to the temperature at the liquid surface and not at the paramagnetic salt (but see below).

As the temperature of $\mathrm{A}$ changed with changing bath pressure, the variation in susceptibility was detected as a change in the bridge setting, $n$. Since the approximate calibration formula of the thermometer was $T=34 / n$ and the precision of bridge setting was $\Delta n=0.001$, temperature changes could be observed as follows: At $4^{\circ} \mathrm{K}, 5 \times 10^{-4} \mathrm{deg}$; at $3^{\circ} \mathrm{K}, 2.5 \times 10^{-4} \mathrm{deg}$; at $2^{\circ} \mathrm{K}, 1.2 \times 10^{-4} \mathrm{deg}$; at $1.3^{\circ} \mathrm{K}, 5 \times 10^{-5} \mathrm{deg}$. This precision was not realized in practice because of small fluctuations at the lowest pressures and to the necessity for applying small corrections for the nonlinearity of the bridge, but the probable error due to such effects was less than 1 millidegree thoughout the range of measurement $\left(1.3^{\circ}\right.$ to $\left.4.2^{\circ} \mathrm{K}\right)$.

Subsequently the apparatus was redesigned, for reasons given below, and the second version is shown in figure $1, \mathrm{~b}$. In this modification there was no vacuum jacket shielding the tube, F, the vaporpressure bulb was shortened considerably, and access to the latter was made possible by winding the coils of the mutual inductance on demountable formers. This assembly proved thoroughly satisfactory from the point of view of rigidity.

\section{Experimental Procedure}

With liquid helium in the cryostat, liquid helium was introduced into the vapor-pressure bulb by condensation of gas under a small overpressure. Prior to the silvering and final assembly, a test filling was carried out to determine the exact quantity of helium required to submerse the crystal, $\mathrm{A}$, and to observe the change of liquid level within the bulb during continuous cooling of the bath. This level will tend to rise because of condensation of the helium gas in the manometer system and fall because of increased density of the liquid as the lambda point is approached. In the present apparatus, the former effect tended to outweigh the latter, and there resulted about a $3-\mathrm{mm}$ submersion at the lambda point. As the precise depth varied with bath level (a large part of the dead-space gas is contained in the section of $\mathrm{F}$ that is immersed), the possible correction of $0.03 \mathrm{~mm}$ of $\mathrm{Hg}$ was not applied to the measured value of $p$. [The corresponding error in $T$ is $3 \times 10^{-4}$ deg at the lambda point and becomes progressively smaller as $T$ increases; below the lambda point there is, of course, no depth correction.]

In an experimental determination of helium vapor pressure the most probable sources of error, i. e., extraneous heat influxes to the bulb, will lead to an overestimate of $p$. Apart from the more familiar radiation and conduction effects, there is one peculiar to low-temperature apparatus; viz., a heat influx due to oscillations in the gas column in a tube such as F. As a result of these heat leaks, the surface temperature of the bulb liquid will be raised, and temperature inhomogeneity will persist because of the low thermal conductivity of the liquid. A thermometer below the surface will therefore be at a lower temperature than that corresponding to the measured value of $p$. The paramagnetic salt is a much better heat conductor than liquid helium, but, even so, a very small heat influx (if all passes through the salt) will suffice to set up a differential of several millidegrees across a 1-in. sphere. (A rough calculation gives: at $4.2^{\circ} \mathrm{K}, 5 \times 10^{-4}$ watt per millidegree; at $2.2^{\circ} \mathrm{K}, 10^{-4}$ watt per millidegree.) The employment of a vacuum jacket in order to 
avoid a cold spot on tube F (fig. 1), was found to do more harm than good; heat flow along $\mathrm{F}$ from above is prevented from entering the bath-liquid, and the conduction heat leak into the vapor-pressure bulb is greatly intensified. A strong manifestation of this effect was observed in the first apparatus, and it was found necessary to admit "exchange gas" into the erstwhile vacuum space, $\mathrm{G}$, in order to minimize this heat leak. [In fact, the "cold-spot hypothesis" appears, upon closer examination, to be invalid for liquid-helium vapor-pressure thermometry in a glass apparatus. This was not appreciated, however, at the time of designing the first apparatus. The hypothesis supposes that a pressure drop exists along the tube corresponding to the full hydrostatic head, which is, for example, $1 \mathrm{~mm}$ of $\mathrm{Hg}$ for a $10-\mathrm{cm}$ depth. (This is equivalent to 1.5 millidegrees at $4^{\circ} \mathrm{K}, 3.7$ millidegrees at $3^{\circ} \mathrm{K}$.) Under such a $\Delta p$, there would be a very large flow of vapor up the tube, which, for the maintenance of the supposed conditions, must cool to the cold-spot temperature and then return downward by convection or condense and flow back as liquid. A rough calculation shows at once that in a tube of the size used (1-cm i. d.) the required heat transfer could not possibly take place through the wall and the process must be self-stifling.]

The presence of gas oscillations in the tube, F, was sought for by connecting a small oil manometer of $2-\mathrm{mm}$ bore between $\mathrm{F}$ and the bath. ${ }^{5}$ None could be detected with the arm connected to the bath closed off. (With this arm open, the oil meniscus oscillated with a period of some 15 sec and an amplitude of about $1 \mathrm{~mm}$.) The same manometer could be used as a direct check on the hydrostatic-head effect. In the first apparatus, the hydrostatic head as measured in this way was always considerably higher than that computed from the level of the liquid in the bath. With the second apparatus, the two values always checked much more closely, which suggests that a heat leak was present in the first apparatus.

The second apparatus was accordingly built without the vacuum shield, G (fig. 1, a) and with smaller holes in the radiation shields (which were also increased in number and more closely spaced). Enough liquid was condensed to reach, initially, the equator of the sphere, $A$, to make better use of the thermal conductivity of the salt as opposed to that of liquid helium.

The manometers were of sufficiently large bore to obviate the necessity for meniscus-height corrections. Thermomolecular pressure effects, and the reflux of helium due to film flow in the $\mathrm{He}$ In region, were negligible for the 1-cm-diameter tube used. Pressures were measured to $0.01 \mathrm{~mm}$ by means of a Wild cathetometer and the readings corrected to standard gravity and $20^{\circ} \mathrm{C}$. Pressures were stabilized through simultaneous adjustment of a finecontrol pumping valve and the current through a small heating coil in the bottom of the cryostat.

${ }^{5}$ We are indebted to J. R. Clement for suggesting this procedure.
The bridge reading, $n$, is a linear function of the susceptibility of the paramagnetic salt specimen, $\chi$, which varies as the inverse of the absolute temperature (Curie law). Departures from the Curie law due to the crystalline field splitting of the ground state spin-quadruplet are negligible down to $1.3^{\circ}$ $\mathrm{K}$, the lower limit of measurement [14]. The calibration formula actually has the form

$$
n-B=A / T,
$$

where $A$ is proportional to the Curie constant of the material, and $B$ is a second constant, equivalent to the bridge balance value at infinite temperature. In order to examine the over-all consistency of a given $p-T$ relation the two constants $A$ and $B$ may be determined by plotting $n$ as a function of $1 / T$ and fitting a straight line to the data. Inserting these values into eq (1), magnetic temperatures, $T_{m}$, are then computed for each measured value of $n$, and the differences $\Delta T=T-T_{m}$ provide a measure of the "over-all validity" of the given $p-T$ relation. [As the latter may be a priori in error in any part of the temperature range investigated, the correct values of $A$ and $B$ are, within limits, infinitely variable and the final choice correspondingly arbitrary. The two constants may be fixed by considering the scale to be correct at any two chosen points, preferably at opposite ends of the temperature interval (cf. Erickson and Roberts [6]).] Values of $\Delta T / T_{m}$ are then plotted against $T_{m}$ for a more sensitive check of the quality of the above fit: for let us suppose that the derived $\Delta T$ 's are entirely due to incorrect choices of the values for $A$ and $B$. Then

$$
\begin{gathered}
\Delta T=T-T_{m}=\frac{\partial T_{m}}{\partial A} \Delta A+\frac{\partial T_{m}}{\partial B} \Delta B \\
=\frac{\Delta A}{A} T_{m}+\frac{\Delta B}{A} T_{m}^{2}, \\
\frac{\Delta T}{T_{m}}=\frac{\Delta A}{A}+\frac{\Delta B}{A} T_{m} .
\end{gathered}
$$

and

Bearing in mind that this type of plot will tend to exaggerate deviations at the lowest temperatures, the best straight line may now be drawn and the originally chosen values of $A$ and $B$ modified accordingly (see footnote 5). By such a procedure it was found possible to assign final values to $A$ and $B$ with confidence that any alternative choices that could be readily permitted would only affect the values of $\Delta T$ in a minor way and would not invalidate the general conclusions as to the over-all correctness of the scale. [This procedure becomes the more acceptable, the better is the $p$ - $T$ relation under examination. In the case of the 1948 Scale, for example, the errors are so large that the choice of $B$ would have to be supported by an independent determination of this quantity from measurements at high temperatures; this was not possible in the present apparatus where the measuring coils were immersed in liquid helium.] 
TABLE 1. Corresponding values of pressure in millimeters of mercury, $p$ (corrected to $20^{\circ} C$ and standard gravity), and bridge dial-setting, $n$ (corrected for nonlinearity in decade scale)

\begin{tabular}{|c|c|c|c|c|c|c|c|c|c|}
\hline \multicolumn{2}{|c|}{ June 7,1955} & \multicolumn{2}{|c|}{ June 8, 1955} & \multicolumn{2}{|c|}{ June 16,1955} & \multicolumn{2}{|c|}{ June 17,1955} & \multicolumn{2}{|c|}{ August 17,1955} \\
\hline$p$ & $n$ & $p$ & $n$ & $p$ & $n$ & $p$ & $p$ & $p$ & $n$ \\
\hline $\begin{array}{l}768.00 \\
751.77 \\
627.49 \\
508.12 \\
341.13\end{array}$ & $\begin{array}{r}\text { 11. } 223_{1} \\
11.178_{3} \\
\text { 10. } 803_{0} \\
\text { 10. } 334_{1} \\
9.4437\end{array}$ & $\begin{array}{c}748.67 \\
413.42 \\
41.060 \\
37.894 \\
23.473\end{array}$ & $\begin{array}{r}11.164 \\
9.8765 \\
3.8058 \\
3.5779 \\
2.1827\end{array}$ & $\begin{array}{l}758.94 \\
758.12 \\
680.15 \\
603.56 \\
501.32\end{array}$ & $\begin{array}{l}11.202_{0} \\
11.2000 \\
10.9778 \\
10.723_{2} \\
10.3174\end{array}$ & $\begin{array}{l}769.97 \\
750.63 \\
550.49 \\
547.46 \\
315.84\end{array}$ & $\begin{array}{r}11.2273 \\
11.173_{5} \\
10.520_{8} \\
10.510_{3} \\
9.258_{5}\end{array}$ & $\begin{array}{l}726.55 \\
726.22 \\
603.46 \\
495.96 \\
320.30\end{array}$ & $\begin{array}{l}\text { 17. } 012_{3} \\
17.013_{2} \\
17.280_{8} \\
17.5737 \\
18.263_{1}\end{array}$ \\
\hline $\begin{array}{r}254.04 \\
153.91 \\
71.02 \\
70.71 \\
26.03\end{array}$ & $\begin{array}{l}\text { 8. } 736_{5} \\
7.473_{0} \\
5.378_{0} \\
5.376_{1} \\
2.478_{5}\end{array}$ & $\begin{array}{r}16.987 \\
13.827 \\
13.581 \\
8.526 \\
\text { 8. } 556\end{array}$ & $\begin{array}{r}1.2404 \\
0.638_{4} \\
.585_{0} \\
-.795_{0} \\
-.788_{5}\end{array}$ & $\begin{array}{l}402.33 \\
173.72 \\
88.05 \\
87.81 \\
14.676\end{array}$ & $\begin{array}{l}9.829_{6} \\
7.794_{0} \\
5.992_{2} \\
5.984_{1} \\
0.814_{7}\end{array}$ & $\begin{array}{r}45.486 \\
36.834 \\
21.691 \\
8.576 \\
3.502\end{array}$ & $\begin{array}{r}4.110_{3} \\
3.492_{2} \\
1.9595 \\
-0.775_{2} \\
-3.5157\end{array}$ & $\begin{array}{c}181.73 \\
96.08 \\
24.595 \\
17.795 \\
13.126\end{array}$ & $\begin{array}{l}19.224_{2} \\
20.385_{0} \\
23.100_{0} \\
23.755_{2} \\
24.371_{7}\end{array}$ \\
\hline $\begin{array}{r}25.80 \\
\\
\end{array}$ & $\begin{array}{c}2.451_{5} \\
\end{array}$ & $\begin{array}{l}\text { 6. } 415 \\
\text { 3. } 907 \\
\text { 2. } 229 \\
1.263\end{array}$ & $\begin{array}{l}-1.650_{8} \\
-3.173_{0} \\
-4.927_{0} \\
-6.7657\end{array}$ & \begin{tabular}{c}
14.774 \\
\hdashline-1
\end{tabular} & .8367 & & & $\begin{array}{l}\text { 9. } 367 \\
6.141 \\
3.770 \\
2.358\end{array}$ & $\begin{array}{l}25.070_{4} \\
25.951_{0} \\
27.0030 \\
28.0109\end{array}$ \\
\hline
\end{tabular}

\section{Results}

The experimental data, i. e., corresponding values of pressure in millimeters of mercury (corrected to $20^{\circ} \mathrm{C}$ and standard gravity) and bridge reading (corrected for nonlinearity), are given in table 1.

\subsection{Comparison With the Clement Equation}

Four runs on different days were made with the first apparatus and the results could be harmonized, using slightly different values for the constants $A$ and $B$, with a quite small scatter. This is shown in fig. 2, a $^{6}$

The signal feature of these results is a large positive deviation hump ( $\Delta T \sim 5$ millidegrees) in the middle of the He I region. The deviations are very small in the He II region (less than 1 millidegree) and achieve significant negative values above $4^{\circ} \mathrm{K}$. From what has been said in the preceding section, the good fit in the He II region could be fortuitous, and one might, for example, reassess the thermometer calibration to give a zero deviation in the region of the boiling point and again somewhere in the middle, say, of the He II region. This has been done in figure $2, \mathrm{~b}$. The main effect is to exaggerate the $3^{\circ} \mathrm{K}$ "hump" while only slightly affecting the points below the lambda point.

On either assessment, however, the suggestion remains that the "Clement temperature" $\left(T_{\mathrm{Cl}}\right)$ is too high by several millidegrees in the neighborhood of $3^{\circ} \mathrm{K}$. Equally well, of course, the measured vapor pressures could be too high here due to errors of measurement, such as would be occasioned by extraneous heat influx to the bulb. All the data of figure 2 were obtained with exchange gas introduced into the jacket, G (fig. 1, a). Without exchange gas the $3^{\circ} \mathrm{K}$ hump became several times larger, and this finds a ready explanation in terms of heat conducted down tube F (fig. 1, a), as discussed in section 3 . By the same token, the hump of figure 2 might be due to an ineradicable heating effect, and that it is partly so is supported by the data obtained with the second apparatus, shown in figure 3.

${ }_{6}^{6}$ The results as presented in figs. $2 \mathrm{a}$ and 3 were actually obtained using the same $A, B$ values as finally chosen for the corresponding Van Dijk-Durieux evaluation (figs. 4 and 5 , respectively). It so happens that these values provide as good a general fit as any others.

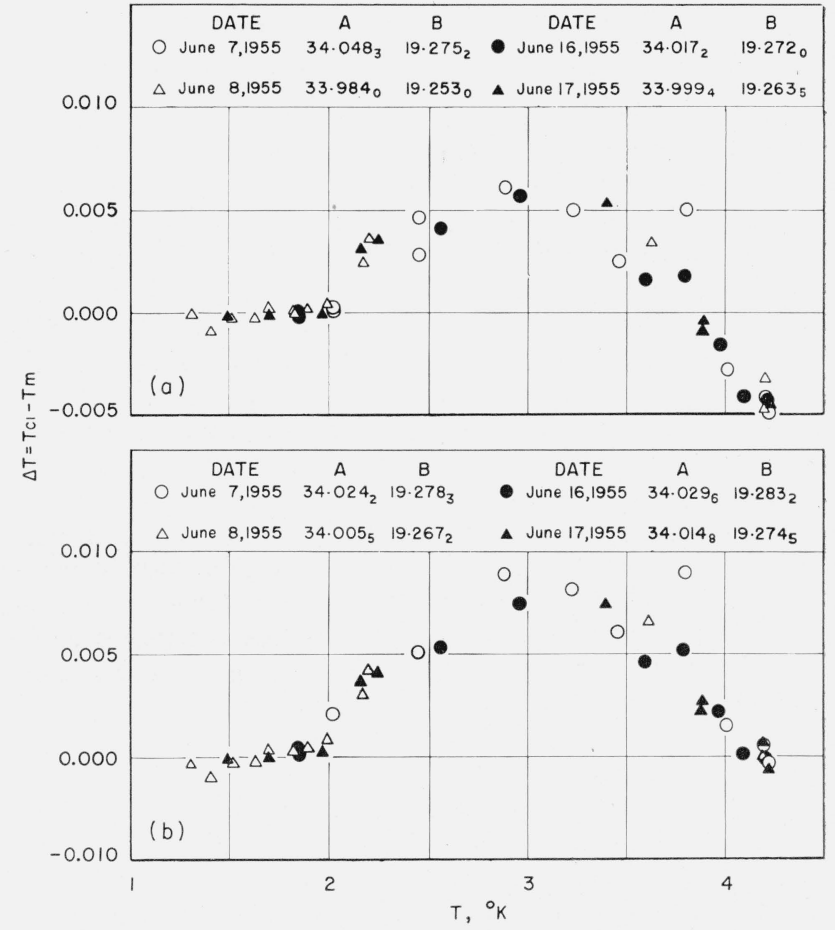

Figure 2. Deviation of temperature as indicated by vapor pressure and Clement $p-T$ relation $\left(T_{\mathrm{C} 1}\right)$ from magnetic temperature $\left(T_{m}\right)$ as a function of $T$.

a, Results of June $1955 ; \mathrm{b}$, data reanalyzed with different values of constants $A$ and $B$, equation (1), to give $\Delta T=0$ at normal boiling point.

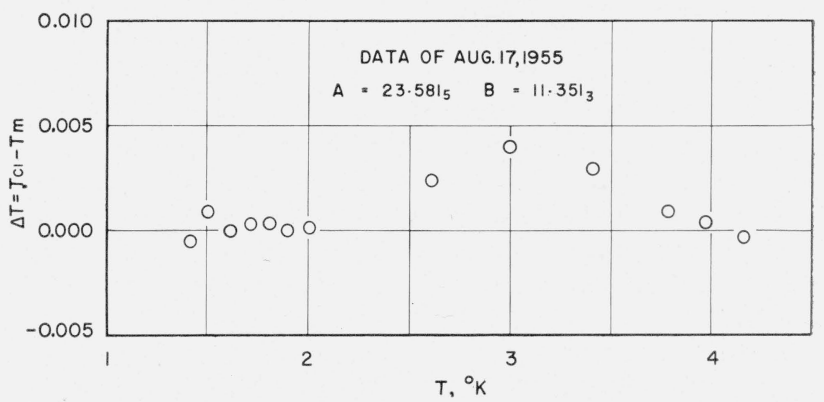

Figure 3. Results of August 1955 analyzed in terms of Clement temperatures, corresponding to June data of figures $2 a$ and $2 b$. 
Only one experiment was carried out with this apparatus, and the data are accordingly somewhat sparse. The thermometer calibration was quite different from the previous case, i. e., one was then operating upon a different section of the bridge windings, which is an advantage in checking the internal consistency of the bridge. The results are very similar to those of figure $2, b$, but the hump in the He I region is much less pronounced (see footnote 6 ).

\subsection{Comparison With the Van Dijk-Durieux Table}

Recently Van Dijk and Durieux (see footnote 3 ) have recalculated the $p-T$ relation for helium, making a reassessment of the best available thermodynamic data. Their results are not in exact accord with the Clement equation and, in fact, deviate from it in the He I region after the manner of the points in figure 3 , with a $\Delta T_{\max }$ of 3.6 millidegrees at $2.9^{\circ} \mathrm{K}$. In the He II region, $\Delta T\left(=T_{\mathrm{C} 1}-T_{\mathrm{VDD}}\right)$ increases steadily from $-0.001 \mathrm{deg}$ at $1.9^{\circ} \mathrm{K}$ to +0.0016 deg at $1.0^{\circ} \mathrm{K}$.

In consequence, the magnetic thermometer data of this report can be fitted somewhat more closely to the Van Dijk-Durieux table than to the Clement equation. Figure 3 has been obtained in a rather subjective manner, with an eye to the general features of a plot of $T_{\mathrm{C} 1}-T_{\mathrm{VDD}}$ versus $T$.

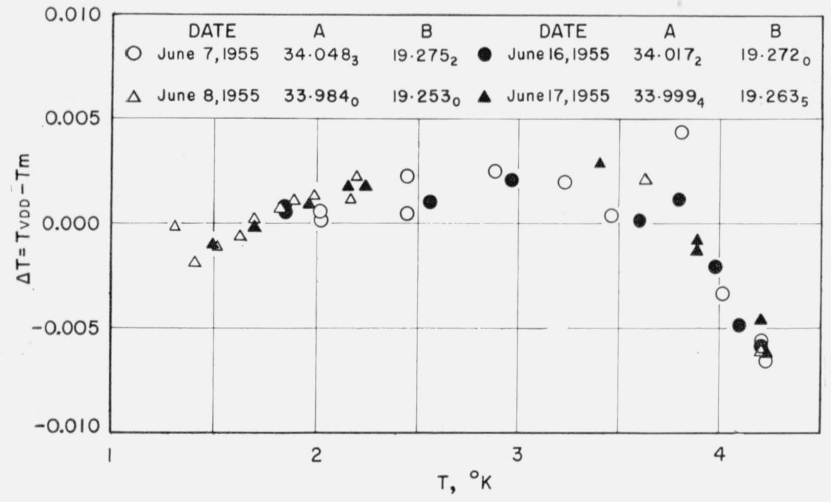

Figure 4. Deviation of temperature as indicaied by vapor pressure and Van Dijk-Durieux $p-T$ relation ( $T_{\mathrm{VDD}}$ ) from magnetic temperature, $T_{m}$, as a function of $T$.

Results of June 1955.

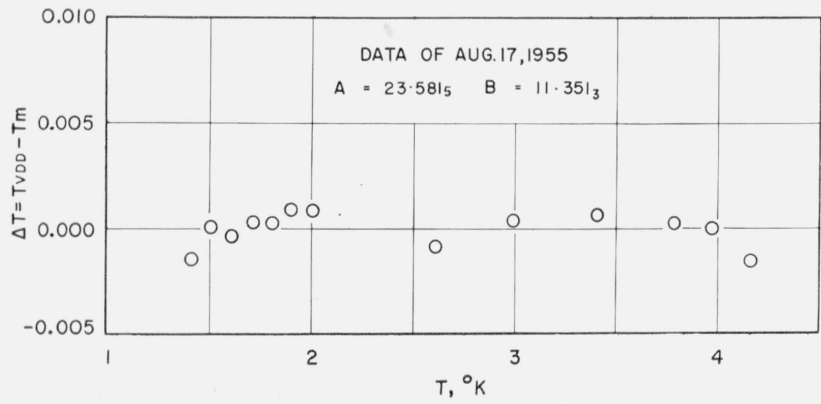

Figure 5. Results of August 1955 analyzed in terms of "Van Dijk-Durieux temperatures," corresponding to June data of figure 4.

(Compare fig. 3.)
The latter [not shown] shows zero deviation at $1.7^{\circ} \mathrm{K}$ and a minimum deviation at about $3.8^{\circ} \mathrm{K}$; these features have been used as a guide in obtaining the calibration curves from which figure 4 is derived. It can be seen that a two-point tie-down is forced on one here, as it is impossible to choose values of $A$ and $B$ (eq (1)) that will give effectively zero $\Delta T$ over a significantly large temperature interval without causing unduly large deviations elsewhere. Thus one may keep the magnitude of $\Delta T\left(=T_{\mathrm{VDD}}-\right.$ $T_{\mathrm{m}}$ small throughout the entire range of measurement but then one also obtains a systematic deviation in the He II region.

The August data are plotted in figure 5, which is to be compared with figure 3 . Figure 5 was obtained as the best fit throughout the entire range of measurement, and it, too, has the feature of practically zero deviation at $1.7^{\circ}$ and $3.8^{\circ} \mathrm{K}$. The magnitude of $\Delta \mathrm{T}$ now lies within \pm 1.5 millidegrees throughout the range of measurement.

\section{Discussion}

The two sets of data, viz., the June results with the first apparatus and the August results with the second, do not agree quantitatively but do show qualitatively the same behavior. It is felt that the August data are the more valid (smaller heat leaks into vapor-pressure bulb), and these agree very closely with the Van Dijk-Durieux calculated $p-T$ table. Both Van Dijk and Clement have recently made significant modifications to their respective $p-T$ tabulations. In a subsequent report the present authors will reevaluate the above data together with the results of a new series of measurements.

None of the present measurements can be brought into very close accord with the Clement equation, in contrast to the results of Erickson and Roberts [6]. The greater part of the latter data was obtained from bath-pressures plus hydrostatic-head correction, which might account for the discrepancy, since the conditions necessary for the exact validity of applying such a correction are impossible to achieve in practice.

The significant differences among the data of Erickson and Roberts and the first and second series reported here point up a major problem in vaporpressure thermometry in the liquid-helium region, viz., the difficulty of reproducing results from one apparatus to another and of checking any $p-T$ relation to the desirable accuracy of 1 millidegree. From the practical point of view, one desires to obtain accurate values of absolute temperature from simply performed measurements of vapor pressure, the latter being an essentially minor part of any given investigation. It is not entirely unreasonable to seek a solution in the direction of making rigid stipulations concerning the technique of vaporpressure measurement and to evolve a "practical $p$ - $T$ relation" that is different from that calculated on a thermodynamic basis.

On the other hand, it is true that the differences between the Clement equation and the Van Dijk- 
Durieux table are never greater than a few millidegrees. Allowing a 2-millidegree precision for the latter table (as its authors claim), a "Clement tomperature" should have a maximum deviation from the thermodynamic temperature of 5.6 millidegrees at $2.9^{\circ} \mathrm{K}$, a probable error that is tolerable for many of the common research problems in this region. Experiments demanding a knowledge of $d T / d p$ (e. g., specific-heat determinations) are, of course, most sensitive to deficiencies in the temperature scale.

The authors are greatly indebted to J. R. Clement of the U. S. Naval Research Laboratory for many stimulating discussions and helpful suggestrons. It is a pleasure to acknowledge the further benefit derived from general discussions of the problem with R. A. Erickson (Ohio State University), M. P. Garfunkel (Westinghouse), P. H. Keesom (Purdue University), W. E. Keller (Los Alamos), L. D. Roberts (Oak Ridge), C. A. Swanson (Iowa State College); and with H. van Dijk, D. de Klerk, and M. P. Durieux (Kamerlingh Ones Laboratory).
6. References

[1] H. van Dijk and D. Shoenberg, Nature 164, 151 (1949).

[2] G. Schmidt and W. H. Keesom, Physica 4, 963, 971 (1937).

[3] B. Bleaney and F. Simon, Trans. Faraday Soc. 35, 1205 (1939).

[4] H. Kamerlingh Ones and S. Weber, Leiden Commun. $147 \mathrm{~b}(1915)$.

[5] J. Kistemaker, Physical 12, 272 (1946).

[6] R. A. Erickson and L. D. Roberts, Phys. Rev. 93, 957 (1954).

[7] R. Burman and C. A. Swenson, Phys. Rev. 95, 311 (1954).

[8] J. R. Clement, J. K. Logan, and J. Gaffney, Phys. Rev. 100, 743 (1955).

[9] W. S. Cork, M. P. Garfunkel, C. B. Satterthwaite, and A. Wexler, Phys. Rev. 98, 1699 (1955).

[10] W. E. Keller, Phys. Rev. 97, 1 (1955); 98, 1571 (1955).

[11] R. P. Hudson, "Temperature, its measurement and controd in science and industry," vol. II, p. 185 (Reinhold Publishing Corp., New York, N. Y., 1935).

[12] P. H. Keesom and N. Pearlman, Phys. Rev. 98, 548 (1955).

[13] D. de Klerk and R. P. Hudson, J. Research NBS 53, 173 (1954) RP 2530.

[14] R. P. Hudson, Phys. Rev. 88, 570 (1952).

Washington, November 22, 1955.

104 